\title{
Multiculturality skills, health care and communication disorders
}

\author{
Competências em multiculturalismo, assistência \\ à saúde e transtornos de comunicação
}

Competencias multiculturales, cuidados en el
ámbito de la salud y problemas de comunicación

Bárbara G. Goulart 1

Rafaela Soares Rech 1

doi: 10.1590/0102-311X00217217

There are 65.6 million people around the world who have been forced from their homes, primarily in the Middle East 1. Large numbers of refugees have also originated from the Democratic Republic of the Congo, the Central African Republic, Myanmar, Eritrea, and Colombia 2. Many of the refugees and immigrants are included in the $20 \%$ of the world's poorest people who have some type of disability ${ }^{3}$. Consequently, there is an increased demand for health care for individuals with diverse language, religious, cultural, and social backgrounds and institutions. Health professionals are being asked to develop a greater knowledge of the needs of these diverse populations ${ }^{4}$. The multiculturality of contemporary life reflects the economic globalization and migration processes. The movement across borders are a result from religious bias, warfare, and the search for a better life. Greater knowledge and respect for the populations from different origins across the world are necessary for health care actions to be more effective 5 . Understanding cultural and language differences is necessary in clinical practice to provide therapeutic strategies that are appropriate to these different groups.

Cultural competence education for health professionals must ensure that all adults and children receive equitable and effective health care, particularly those from culturally and linguistically diverse backgrounds 6. Cultural competence is an important strategy to address health inequalities. Being aware of the processes of interculturality of contemporary life is necessary: the increase on the number of immigrants and refugees, understanding the structure of various languages to differentiate a typicality from disorders, and the use of the internet facilitates the knowledge development of health practitioners. A clinical speech-language pathologist must be prepared to care for patients from different contexts. To address this demand, researchers in human communication sciences must go beyond the traditional approaches and investigate diverse strategies related to speech-language pathology assessment and intervention.

International collaboration is being developed as a strategy to provide services to underserved and unserved populations with disorders 7 . Collaboration involves a team that provides a diverse set of skills, providing ideal approaches to a population that requires support. In collaborations, those with expertise transfer their knowledge to those who need it. One example is the collaboration in Guangzhou, China 8. The Bureau of Education of Guangzhou sponsored a three-year grant in 2016 to offer continuous training to special education teachers. The unserved population that was targeted were children with autism spectrum disorders (ASD). Results of the collaboration were the greater knowledge of strategies, interventions, and resources for sensory processing disorders of parents
${ }^{1}$ Universidade Federal do Rio Grande do Sul, Porto Alegre, Brasil. ${ }^{2}$ Lehman College, City University of New York, New York, U.S.A.

Correspondence

B. G. Goulart

Universidade Federal do

Rio Grande do Sul.

Rua Ramiro Barcelos 2600,

Porto Alegre, RS 90035-003, Brasil.

bngoulart@gmail.com 
and teachers; an understanding of the environmental factors that may impact on a child's behavior; sensory factors that may impact children's focus on tasks in a classroom, visual and auditory aids for children who would benefit from these supports, the social/emotional needs of children, and the environmental factors that may impact on children's behaviors.

Society is constantly changing, thus, professionals must seek knowledge of the specific theoretical and practical assessment and intervention strategies that best benefit children and adults. Professionals must also update their knowledge consistently. This is a responsibility as a citizen and as a health professional. Cultural diversity requires understanding ethnic, gender, beliefs, and religious issues, along with socioeconomic and cultural conditions. The interdisciplinarity between health, culture, and communication implies new paradigms and strategic challenges (political, theoretical, scientific, educational and clinical practices). Organizational discussions and articulations are essential to improve the promotion of individual and collective health for native populations or migrants. The intercultural relationship allows a holistic health care, promoting the pluralism of well-being.

In the academic context, it is important that professors and students discuss aspects related to cultural diversity and encourage integrative practices based on the principles of interculturality. In addition, encouraging professors, students, and health practitioners to expand their vision and ideas is important. A study found that students who had taken a course in bilingualism were better able to distinguish language differences from disorders and had better knowledge of linguistic and dialect differences than participants who had not taken this type of course ${ }^{9}$. Professors may encourage students to engage in collaboration to exchange knowledge with other professionals and families across the globe and make students aware of differences in foreign languages. Both professors and students may use the internet to understand the health disorders in refugees and immigrants, in addition to reading international scientific articles to gain greater knowledge.

In the clinical context, it is essential that the professionals defuse their internal prejudices and act in a universal manner. Having empathy, accepting different thoughts, and adapting their knowledge to each adult or child who requires support is critical. Understanding that diagnosis and rehabilitation are not isolated is essential, since different social contexts interfere in these aspects to some extent. The exchange of knowledge with professionals from other areas and in other countries is important for a culturally expanded training.

Finally, it is necessary that speech-language therapists and other health professionals understand the importance of employing clinical practice strategies that consider different cultures. Engaging in discussions about this subject to consider the experiences and knowledge of other professionals is also important to ensure a successful training. Those with health disorders are not only refugees and immigrants, many are within our own countries and may lack health services, this should always be considered. These are the underserved and unserved across the globe. 


\section{Contributors}

B. G. Goulart, S. Levey, and R. S. Rech participated in the idealization, analysis of references and writing of the manuscript.
1. United Nations High Commissioner for Refugees. Figures at a glance. http://www.unhcr. org/figures-at-a-glance.html (accessed on 24/ Oct/2017).

2. United Nations High Commissioner for Refugees. Global trends: forced displacement in 2015. http://www.unhcr.org/576408cd7.pdf (accessed on 24/Oct/2017).

3. Disabled World. Definition: defining the meaning of annual disability status report 2016 http://www.disabled-world.com/disability/ statistics/ (accessed on 24/Oct/2017).

4. Morales-Moreno I, Giménez-Fernández $\mathrm{M}$, Echevarría-Pérez P. The cultural dialogue on the domestic dimension of care to immigrant caregivers in Spain. Rev Latinoam Enferm $2015 ; 23: 821-8$

5. Horvat L, Horey D, Romios P, Kis-Rigo J. Cultural competence education for health professionals. Cochrane Database Syst Rev 2014; (5):CD00940.

6. Masland MC, Kang SH, Ma Y. Association between limited English proficiency and understanding prescription labels among five ethnic groups in California. Ethn Health 2011; 16:125-44.

7. Jackson SC, Bridges-Bond S, Cromartie J, Blankson E. Collaboration with caregivers and educators in the Dominican Republic: promoting children's language and literacy development. Perspect ASHA Spec Interest Groups 2017; 2:50-60.

8. Cheng LRL, Levey S. People with disabilities: the underserved and unserved. Med Res Arch 2016; 4:1-15.

9. Levey S, Sola J. Speech-language pathology students' awareness of differences versus disorders. Contemp Issues Commun Sci Disord 2013; 40:8-14. 